\title{
XPS Spectromicroscopy as an Optimised Technique for Materials Characterisation.
}

\author{
D. Surman ${ }^{2}$, C. Moffitt ${ }^{2}$, A. J. Roberts ${ }^{1}$, S. J. Coultas ${ }^{1}$, J. D. P. Counsell ${ }^{1}$ and C. J. Blomfield ${ }^{1}$ \\ 1. Kratos Analytical Ltd, Wharfside, Trafford Wharf Road, Manchester, M17 1GP, UK \\ 2. Kratos Analytical Inc., 100 Red Schoolhouse Road, Chestnut Ridge, NY
}

X-ray Photoelectron Spectroscopy (XPS) is widely used in determining surface chemistry of materials. Improvements in instrument sensitivity mean that spectra are routinely acquired from areas with diameters in the tens of microns, although most routine analysis is performed at much larger areas. The assumption is that the material and spectra are homogeneous over the area being probed is often made although it may not be true. Information on the lateral distribution of elemental and chemical states on a surface can be probed using XPS imaging either at a single binding (kinetic) energy or over a narrow energy range corresponding to a core-level photoemission peak.

Multispectral XPS imaging, also referred to as Spectromicroscopy, where a series of images incremented in energy such that each pixel contains a spectrum, is relatively new and under exploited technique for surface characterization. An advantage of Spectromicroscopy is that spectral information can be reconstructed from defined areas which are smaller than those possible with focused x-ray or virtual probe selected area XPS. This means that the reconstructed spectra are no longer averaged over the total area from which the image is acquired such that both sample and instrument dependent differences can be studied.

XPS images are acquired using a $256 \times 256$ pixel array to maximize the information content and minimize the potential analytical analysis area. The $256 \times 256$ pixel multi-spectra image contains $>65,500$ spectra which is ideally suited to multivariate analysis. Chemically resolved images, Fig. 1 shows the distribution of Fluorine following the functionalization of a polymer surface, can be obtained in a few seconds such that an entire analysis can be completed in minutes. The acquisition of multiple elemental/chemical images can lead to a complete characterization of a surface. The data however is frequently noisy and often requires additional processing.

Development of data processing methods to support Spectromicroscopy data reduction has been necessary and a number of approaches have been successfully applied in the characterization of model and realworld samples [1-3]. Multivariate analysis can be used to classify regions of interest across the field of view and data can be partitioned such that chemical state; changes in peak position and background shape can be investigated. Here we detail the use of Spectromicroscopy for the characterization of complex materials. This approach has enabled the considerable challenges of surface analysis of such materials to be addressed and has allowed the influence of signal from the substrate material to be removed from the samples of interest. Figure 2 shows an example of before and after image processing to significantly improve the information content and quality. Advanced image processing techniques including MV data manipulation will be discussed for improving the information yield of imaging and Spectromicroscopy. 


\section{References:}

[1] E.F. Smith, D. Briggs and N. Fairley, Surf. Interface Anal. 2005, 38, 69-75

[2] J. Walton, N. Fairley, Surf. Interface Anal. 2008, 40, 478 - 481

[3] A.J. Barlow, O. Scott, N. Sano and P.J. Cumpson, Surf. Interface Anal. 2015, 47, 173-175

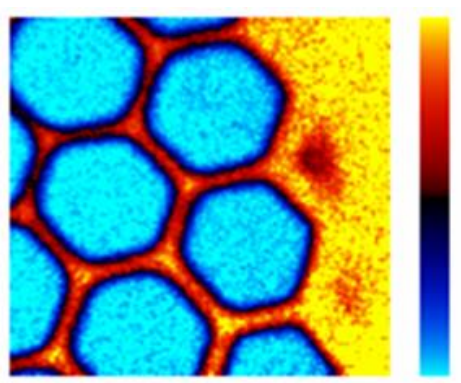

Fig 1. Chemical state image of a Fluorine functionalized polymer surface
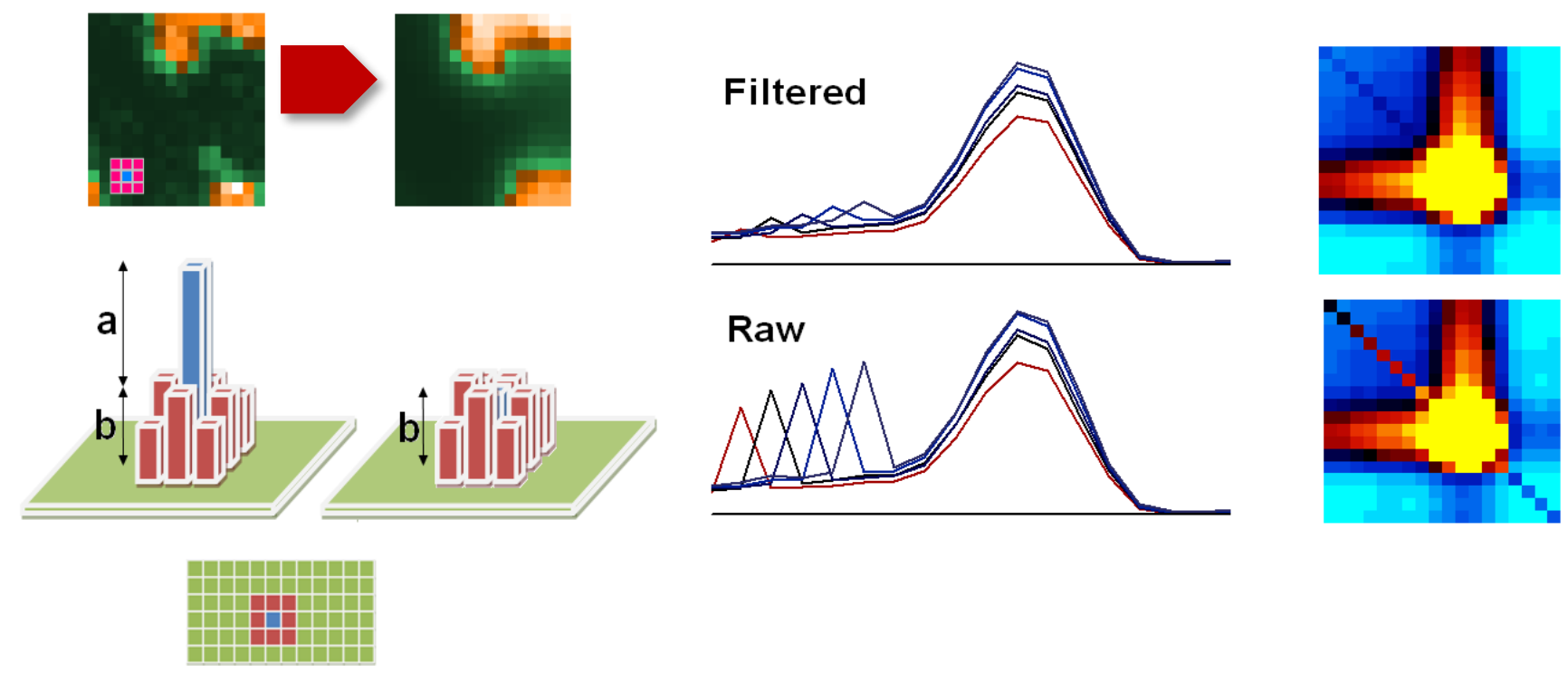

Fig 2. Advanced data treatment for improving image information 